\title{
The use of spectroscopy methods for structural analysis of CVD diamond films, polycrystalline and single-crystal diamonds
}

\author{
Nikolay Ivanovich Polushin ${ }^{1, *}$, Alexander Ivanovich Laptev ${ }^{1}$, Mariya Stanislavovna \\ Shitareva ${ }^{1}$, Dmitry Sergeevich Muratov ${ }^{1}$, Anatoly Lvovich Maslov ${ }^{1}$, Alexey Nikolaevich \\ Kirichenko $^{2}$, Sergey Alexeevich Perfilov ${ }^{2}$, and Tatiana Vladimirovna Martynova ${ }^{1}$ \\ ${ }^{1}$ Research Laboratory of Superhard Materials, National University of Science and Technology MISIS, \\ 119049, Leninsky avenue, 4, Moscow, Russia \\ ${ }^{2}$ Federal State Budgetary Institution "Technological Institute For Superhard and Novel Carbon \\ Materials" FSBI TISNCM, 108840, 7a Tsentralnaya street, Troitsk, Moscow, Russia
}

\begin{abstract}
For the work results correct interpretation, it is important to study initial materials that scientists have to deal with. Currently, there are a large number of different diamond substrates. Comparison of materials among themselves allows you to determine which material you are dealing with. In this work, the methods of infrared (IR) spectrometry, Raman spectroscopy and spectrophotometry are used to study four types of diamond materials: diamond polycrystalline CVD-films; natural singlecrystal diamonds; synthetic polycrystalline HPHT-diamonds (such as DSPC - diamond synthetic polycrystal by GOST 9206-80); polycrystalline CVD-diamonds CDM manufactured by E6. In work it was shown that the Raman spectroscopy allows to measure the effect of heat treatment on changes in the diamond structure, even if it is such highly advanced diamond materials as natural diamonds. Heat treatment affects the perfection of diamond crystal structure by reducing stresses and the number of defects in it due to graphitization process. The IR spectrometry method is effective for determining the shape and amount of nitrogen inclusions in diamond structure. To study polycrystalline CVD-films, the spectrophotometry method turned out to be the most effective, because it made possible to determine a small number of nitrogen defects and draw conclusions about the quality of the films. The investigation of polycrystalline diamonds CDM and DSPC demonstrated that, despite their coarse-crystalline structure, diamond crystallites consist of a highly defective diamond phase; in addition, DSPC-diamonds were studied using this method in the first time.
\end{abstract}

\section{Introduction}

Synthesis of macroscopic size of diamond from the gas phase (CVD-process) occurs at relatively low deposition rates that for polycrystalline diamond are typically $\sim 1-10 \mu \mathrm{m}$.

*Corresponding author: almaslov@misis.ru 
This method was first developed by Soviet researchers [1 - 2]. Both single-crystal and polycrystalline CVD diamond wafers and films are widely used today [3 - 4]. From an economic point of view, it is beneficial to use CVD diamond products in the form of thin micro- and nanocrystalline films with a thickness of several micrometers and less. An important task is to obtain continuous homogeneous films with minimum thickness. The most common microcrystalline diamond films have a columnar structure with a crosswise grain size from one to hundreds of microns depending on film thickness. The result is a significantly lower surface roughness of nanocristalline films than microcrystalline diamond of the same thickness, which, in turn, may be preferred for optics and tribology applications.

It is possible to grow diamond films and wafers with a thickness from fractions of a micrometer to several millimeters with a grain size from 5-10 nm to several hundred microns on substrates of various materials by chemical vapor deposition [5 - 10]. To stimulate the growth of diamond, nucleus were placed on the diamond surface. The solution to the problem of obtaining a thin obtain continuous layer is to increase the density of nucleation that is the average number of nucleus per unit area of the substrate. Spontaneous formation of diamond during its deposition from the gas phase is very-low-probably process due to the high surface energy of the diamond facets. The main effect is achieved due to the chemical growth of existing crystallite nucleus.

From the standpoint of solid state physics, diamond as well as germanium and silicon is a high-energy-gap semiconductor; therefore, optical processes in it are determined by impurities and defects in its crystal structure. Possible impurities and defects in natural diamonds are extremely numerous and very diverse. About 300 optically active centers were found in diamonds, including about 20 varieties of nitrogen defects and about 10 types of intrinsic defects. These centers correspond to more than 2000 bands in the absorption spectra, luminescence and cathodoluminescence spectra, which are only partially deciphered [11 - 13]. Impurities and defects in diamond belong to deep centers, and their optical activity is usually caused by intracenter transitions. Owing to the high symmetry, diamond has no internal single-phonon absorption, but intrinsic or impurity defects lead to a local decrease in symmetry and the appearance of single-phonon absorption.

The main impurity in diamond crystals is nitrogen [6 - 7]. Its concentration can be up to 0.2 atomic percent. Capture of nitrogen atoms by diamond occurs during the crystal formation process. Depending on the temperature and time conditions, they can form various defects in the crystal structure: A, B1, C, B2, and N3. These defects are optically active, that is, they are noticeable in the absorption or luminescence spectra.

An analysis of the typomorphic diamond characteristics is usually carried out with the identification of their morphological and physical characteristics, individualizing both particular crystals and collections from various sources. The choice of research methodology is based on several principles, the main of which is the principle of nondestructive effects in the study of crystals. It is important that the chosen research method does not affect the object of study, does not destroy it, does not change its composition, structure or individual properties, in addition to this it should not require complicated preparation for its implementation. Optical spectroscopy methods allow to examine samples without their destroying. In addition, the advantage of this method is the ability to obtain quantitative results. Therefore, the main methods often used in researches are method of infrared (IR) spectroscopy, which allows one to determine the concentration of defects in diamond structure, and the Raman spectroscopy method, which makes possible to assess the degree of structural perfection of diamond material. Figure 1 [14] demonstrates the Raman spectra for single-crystal diamond and nanostructured CVD-diamond, which confirm that this method is a powerful research tool for assessing the characteristics of the diamond structure. 


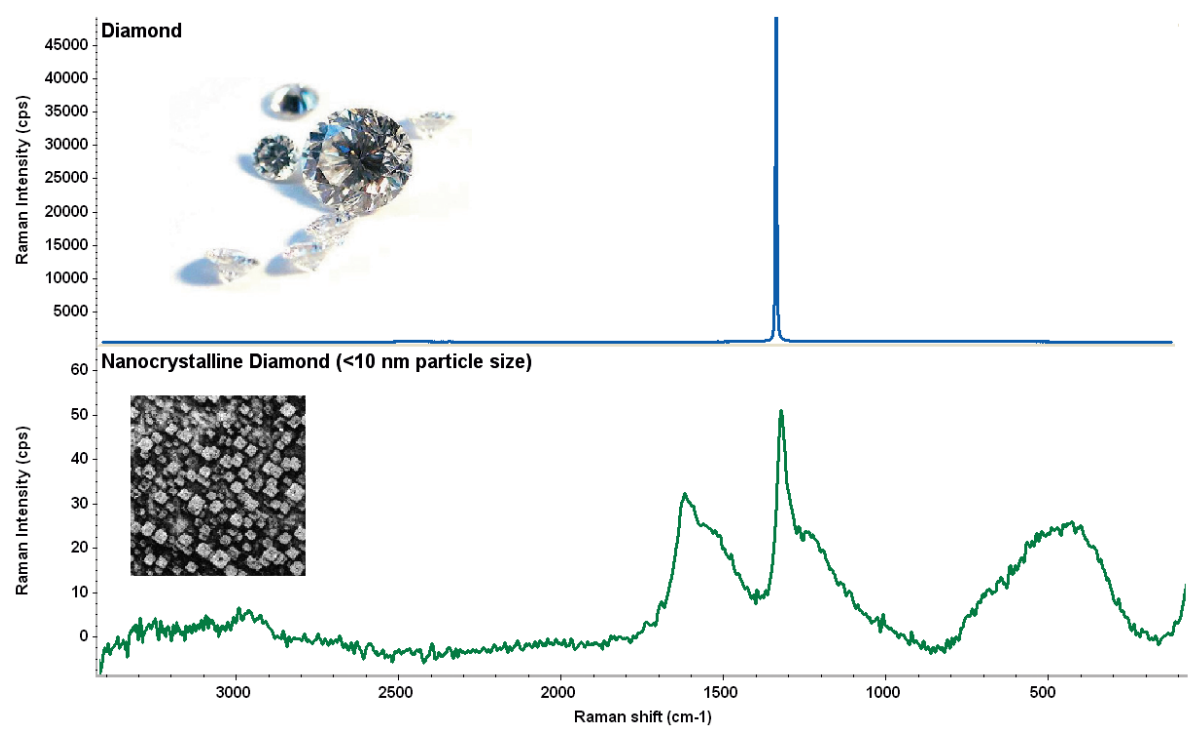

Fig. 1. Raman spectrum of single- and nanocrystalline diamonds [14].

In this work, four types of diamond materials were investigated: polycrystalline diamond CVD-films; natural single-crystal diamonds of group 26a before and after heat treatment; synthetic polycrystalline HPHT-diamonds of the DSPC type [15 - 17]; and polycrystalline CVD-diamonds CDM manufactured by E6 company. Heat treatment of natural single-crystal diamonds was carried out in order to detect changes in the crystal lattice, to assess the selectivity of used spectroscopic methods and to calculate the concentration of defects. For this, we used the methods of infrared (IR) spectrometry, Raman spectroscopy and spectrophotometry.

The IR spectrometry method is based on the selective absorption of electromagnetic emission in the infrared range at frequencies that correspond to oscillation energies in crystals. The set of such frequencies and the corresponding absorption coefficients are individual for each compound and crystalline substance and depend on their composition, structure, symmetry, and perfection of their crystal structure. Therefore, IR spectrometry is widely used to study minerals at the present time. The basis for determining the concentration of defects in diamonds from the absorption spectra in the infrared range is based on the experimentally established fact of the stability of the shape of the absorption bands induced by the main defects. The general outline of the absorption spectrum of diamonds in the single-phonon area is a superposition of the spectra induced by each defects.

Raman spectroscopy is most sensitive to covalent bonds with high symmetry and little or no dipole moment. Carbon-to-carbon bonds fully comply with this criterion; therefore, the Raman spectroscopy allows to detect the smallest changes in the structure of the material. Carbon materials, including nanostructured, consist merely of carbon atoms and they are polymorphic modifications. These include not only diamond and graphite, but also fullerenes, graphene, and more complicated structures, such as nanotubes. All these materials are formed only by carbon-carbon bonds, the spatial orientation of which is different in each material; therefore, we need for control a method sensitive to small changes in the orientation of non-polar bonds. Raman spectroscopy is suitable for studying the structure of carbon materials. Each band in the spectrum corresponds to a specific oscillation of the molecule and its frequency is extremely sensitive to the spatial orientation of the bonds and the mass of atoms (Figure 1). 
This sensitivity of the spectral method provides an opportunity to use it in the analysis of diamonds: the spectra of natural, enhanced and synthetic single crystals differ significantly. The appearance of rather intense bands and amorphous carbon in the spectra of artificial diamonds makes it possible to reliably distinguish them from natural ones; in addition, a specific group of bands in the range of $530-400 \mathrm{~cm}^{-1}$ is present in the spectra of enhanced diamonds. The method can be effectively used to analyze the crystalline perfection of polycrystalline diamond CVD-films and single-crystal diamonds.

It should be considered that the line intensity in CVD diamond films decreases with grain size reduction up to $10 \mathrm{~nm}$. The line intensity in CVD diamond films depends on its growth temperature: it declines with decreasing growth temperature, it disappears completely with a corresponding decrease in temperature. The position of the diamond line can vary significantly depending on the perfection of the diamond sample. In natural diamonds of different origin these lines can be found in the range from 1331 to $1346 \mathrm{~cm}^{-1}$. It is important for diamond studies that the spectral position of the Raman line of the diamond (Raman line) does not change with a change in the excitation wavelength from 244 to $780 \mathrm{~nm}$.

Spectrophotometry allows one to obtain the dependence of the intensity of absorption of impinging light on the wavelength. The method allows to record the absorption spectra of diamonds with nitrogen N3- and C-types defects.

Consequently, these spectroscopy methods are simple, reliable and rapid techniques for studying diamond materials with high reproducibility. Also, the spectrum processing technique based on the results of studying different parts of single-crystals and diamond polycrystalline CVD-films allows us to determine the resulting effect of heat treatment on the crystal structure.

\section{Materials and methods}

Samples for analysis:

1. Natural diamonds of $26 \mathrm{~b}$ group: initial (M1); heat treated (M2). Heat treatment was carried out at $1500{ }^{\circ} \mathrm{C}$ in vacuum for $15 \mathrm{~min}$.

2. Diamond polycrystalline CVD-plates: plate 1 and plate 2 .

3. Synthetic polycrystalline HPHT-diamonds of the DSPC type (synthesis parameters: pressure $8.0 \mathrm{GPa}$, temperature $1900 \mathrm{~K}$, catalyst - nickel).

4. Polycrystalline CVD-diamonds CDM manufactured by E6.

Equipment used in the research: IR-spectrometer Nicolet Thermo, spectrophotometer Cary 400 UV-Vis Spectrophotometr, Raman spectrometer Renishaw inVia Raman Microscope, Raman confocal microscope Thermo DXR.

\section{Results}

As a result of the study of diamond samples, we revealed changes in the crystal lattice depending on the heat treatment and calculated the concentration of nitrogen defects.

The basis for calculating the defect concentration in diamonds from the absorption spectra in the IR-range is based on the fact of the stability of the shape of the absorption bands induced by the main defects established experimentally. Figure 2 shows the IRspectra for the analysed samples. 


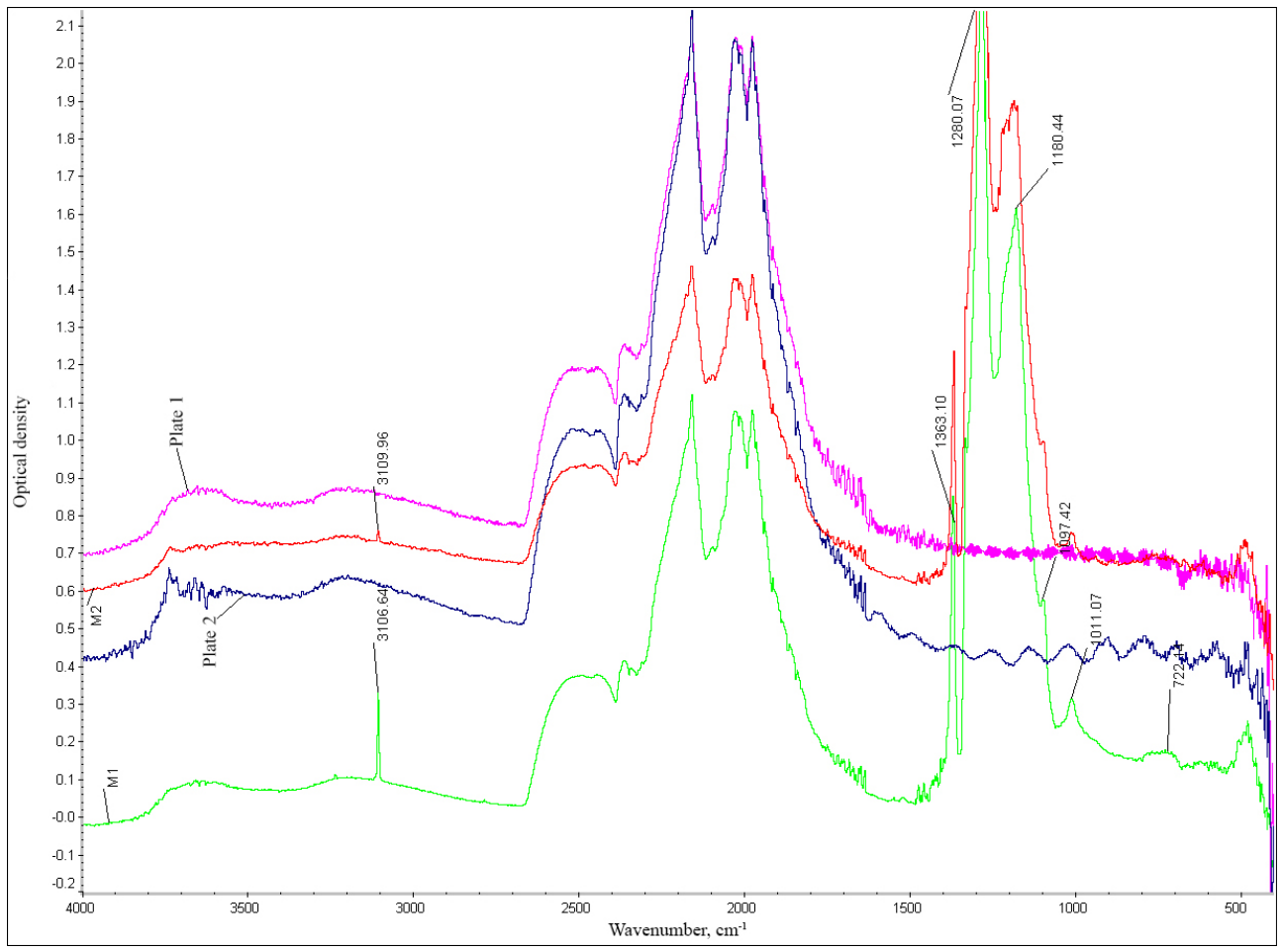

Fig. 2. IR-spectra for natural single-crystal diamonds and polycrystalline diamond CVD-films.

The analysis for samples $\mathrm{M} 1$ and $\mathrm{M} 2$ revealed the type of diamond $\mathrm{AB}$ (the main form of nitrogen is in the form of defect B). Lines 1363.10 and $1180.44 \mathrm{~cm}^{-1}$ response for Bcenters in these samples. The difference between IR-spectra of samples M1 and M2 was detected in the hydrogen line $\left(3106.64 \mathrm{~cm}^{-1}\right.$ for M1 and $3109.96 \mathrm{~cm}^{-1}$ for M2), which indicates the effect of heat treatment on the diamond structure, because the material graphitized after heat treatment.

The calculated concentrations of nitrogen defects were 692 ppm for M1 and $546 \mathrm{ppm}$ for M2.

In the analysis of polycrystalline diamond CVD-films, strong interference is observed due to the small thickness; therefore, an additional analysis was carried out in the visible light range (Figure 3). 


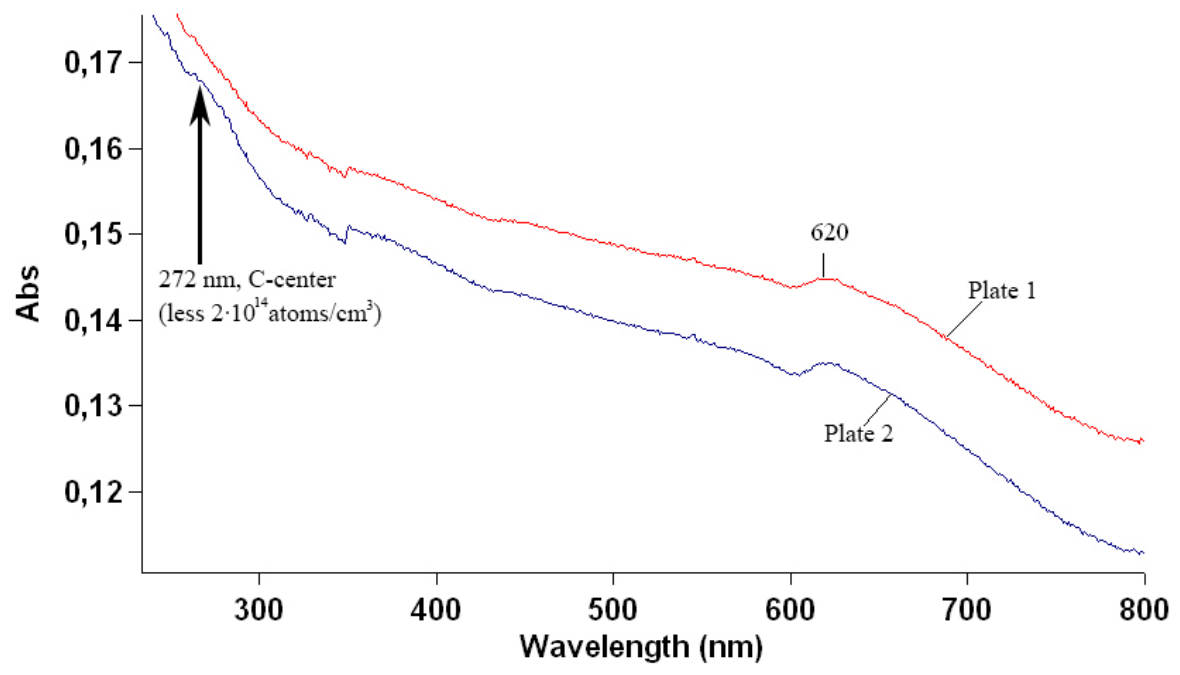

Fig. 3. Spectrophotometry results of polycrystalline diamond CVD-films.

As a result of the analysis by spectrophotometry, a small difference in the thickness of the films was revealed, which is evident from the difference in the arrangement of spectral lines. C-center defects (single nitrogen atoms) were beyond the limits of detection; that indicates about their very small number in the samples and high quality of the analyzed material.

Figures 4 and 5 represents the results of Raman spectroscopy at different wavelength ranges for natural diamond samples M1 and M2. This method made it possible to determine the effect of heat treatment on the crystal structure more accurately.

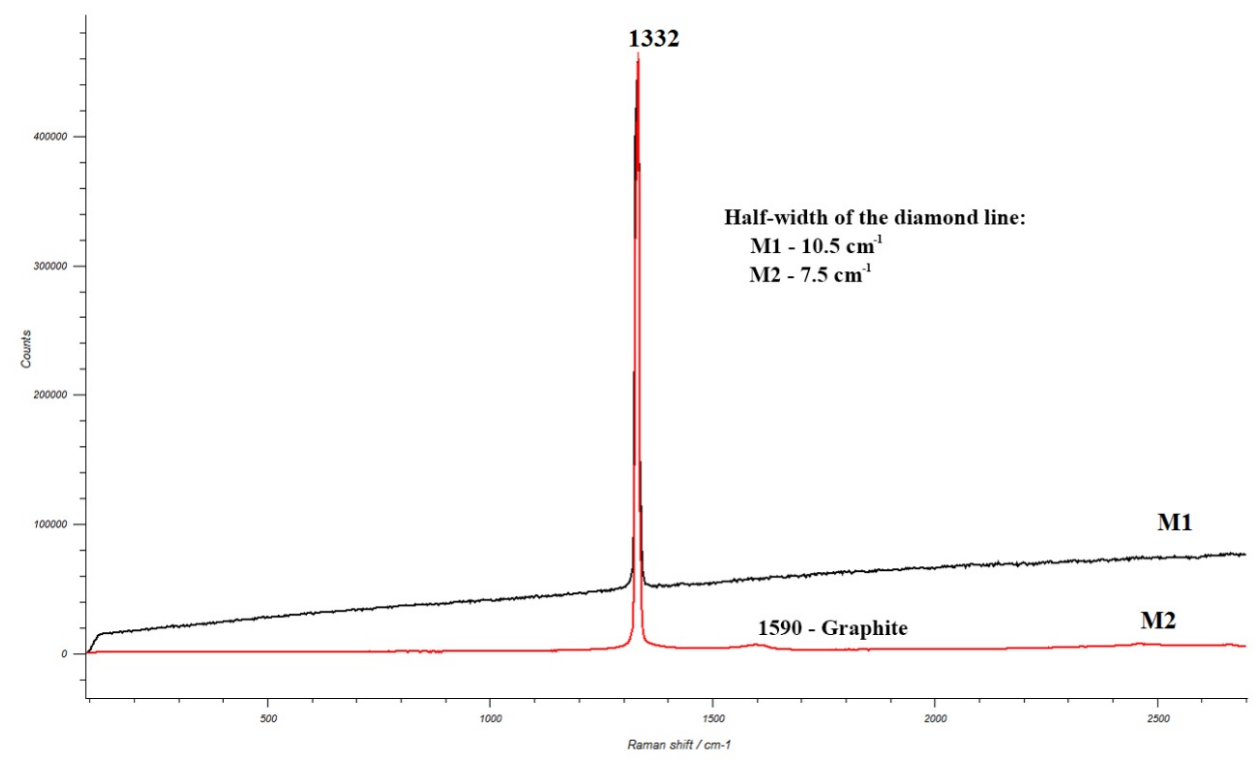

Fig. 4. Raman spectroscopy for natural diamond samples M1 at different wavelength ranges. 


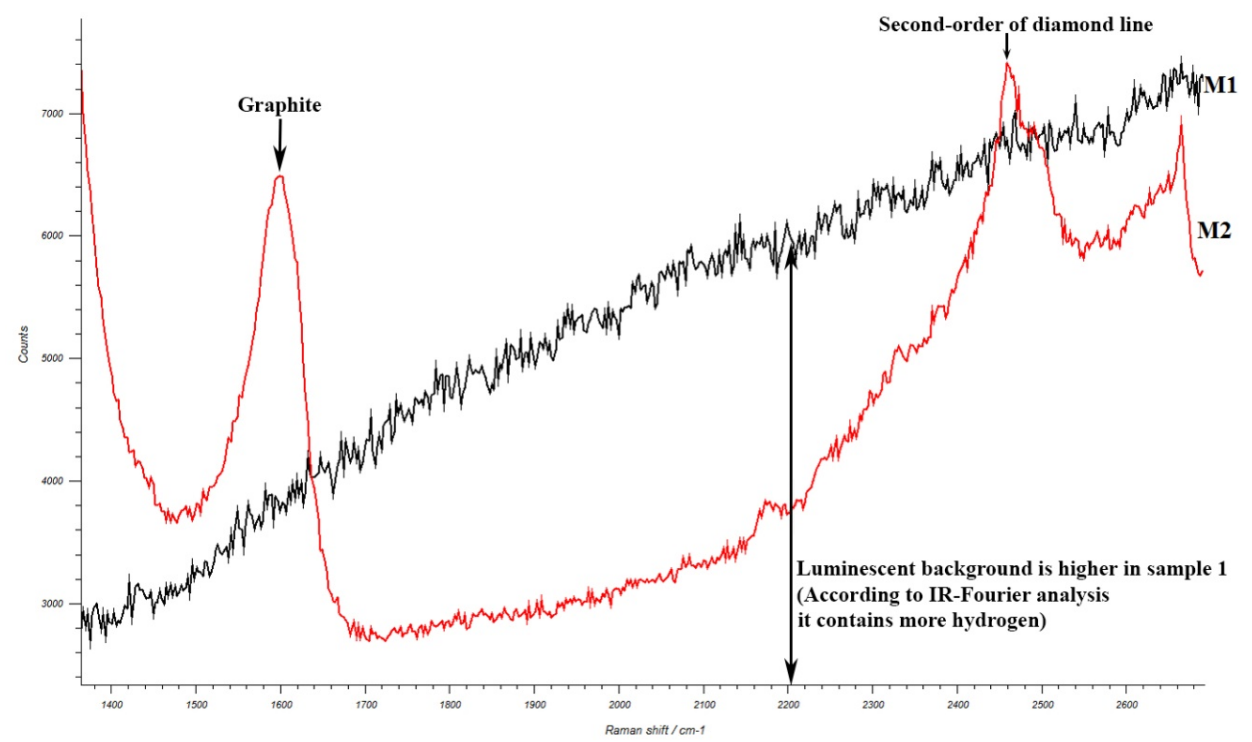

Fig. 5. Raman spectroscopy for natural diamond samples M2 at different wavelength ranges.

Diamond lines in the samples are completely the same. As a result of the analysis of the obtained spectra, it was concluded that the diamond line of the heat-treated sample M2 is narrower than diamond line of sample M1: it is $7.5 \mathrm{~cm}^{-1}$, while for M1 it is $10.5 \mathrm{~cm}^{-1}$. Results indicate an increase in the structural perfection of the heat-treated diamond sample M2 in comparison with the non-heat-treated sample M1. Consequently, heat treatment affects the perfection of the crystal structure of diamond by reducing stresses and the number of defects due to the graphitization process.

Figures 6 and 7 show the results of Raman spectroscopy for CDM and DSPC samples. From these spectra it follows that these diamond materials are characterized by the presence of diamond phase with a large number of defects; the volumetric content of the diamond phase for sample CDM is more than $99 \%$, and for samples DSPC - more than $80 \%$.

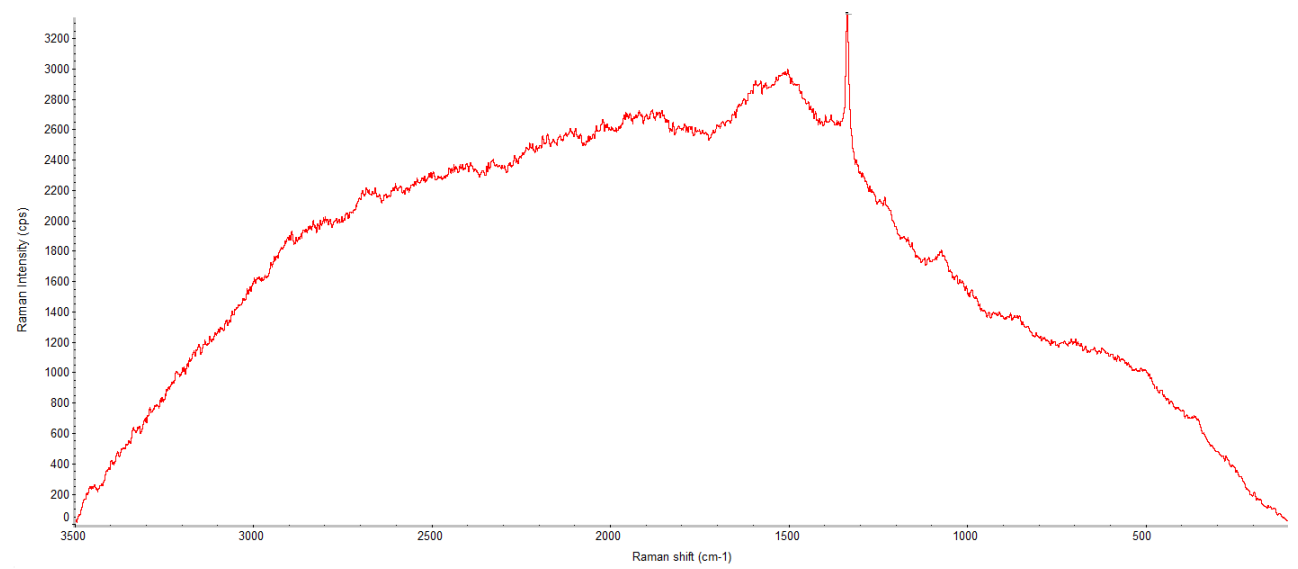

Fig. 6. Raman spectroscopy results for DSPC. 


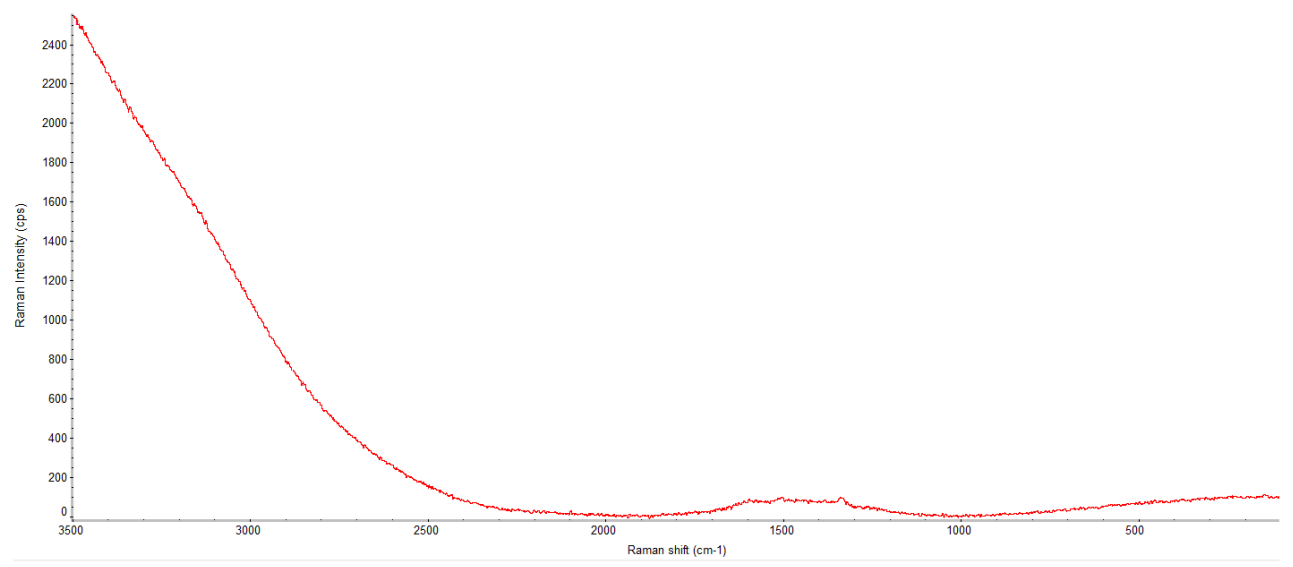

Fig. 7. Raman spectroscopy results for CDM.

\section{Conclusions}

In the study of diamond materials, it was found that the complex of spectroscopic methods used in this work is simple, reliable and rapid method for the analysis of diamond materials with a high degree of reproducibility.

Raman spectroscopy allows to measure the effect of heat treatment on changes in the diamond structure, even if it is such highly advanced diamond materials as natural diamonds. It was found that after heat treatment lines corresponding to the phase of graphite appear and the degree of perfection of the diamond phase increases. Heat treatment affects the perfection of diamond crystal structure by reducing stresses and the number of defects in it due to graphitization process.

As a result of polycrystalline CVD-films studying, it was found that due to their small thickness their analysis by IR and Raman spectroscopy is fraughted with great difficulties; therefore, the spectrophotometry method turned out to be the most effective and this method made it possible to determine a small number of nitrogen defects and draw conclusions about the quality of the studied films.

The investigation of polycrystalline diamonds CDM and DSPC demonstrated that, despite their coarse-crystalline structure, diamond crystallites consist of a highly defective diamond phase; in addition, DSPC-diamonds were studied using this method in the first time.

The IR spectroscopy method is effective and reliable for determining the total content and shape of nitrogen inclusions in CVD-diamond structure.

This research was funded by Ministry of Education and Science of the Russian Federation, grant number 075-15-2019-255 dated July 9, 2019, unique identifier of the agreement RFMEFI57818X0266.

\section{References}

1. B.V. Spitsyn, B.V. Deryagin, Patent USSR №339134. Priority from 10.07.1956 г., published № 17, 1980, 323.

2. B.V. Spitsyn, A.E. Aleksenko, Metal protection, 43, 5, 456-474 (2007)

3. R. S. (Ed.) Sussmann, CVD Diamond for Electronic Devices and Sensors. Chichester: J. Wiley (2009) 
4. R.A. Hmelnitsky, N.H. Talipov, G.V. Chucheva, Synthetic diamond for electronics and optics, 228 (2017)

5. C.J. Chu, R.H. Hauge, J.L. Margrave, Applied Physics Letters, 61, 1393-1395 (1992)

6. M.A. Lobaev, A.M. Gorbachev, S.A. Bogdanov, A.L. Vikharev, D.B. Radishev, V.A. Isaev, V.V. Chernov, M.N. Drozdov, Diamond and Related Materials, 72, 1-6 (2017)

7. R. S. Balmer, J.R. Brandon, S.L. Clewes, H.K. Dhillon, J.M. Dodson, I. Friel, P.N. Inglis, T.D. Madgwick, M.L. Markham, T.P. Mollart, N. Perkins, G.A. Scarsbrook, D.J. Twitchen, A.J. Whitehead, J.J. Wilman, S.M. Woollard, Journal of Physics Condensed Matter, 21, №26 (2009)

8. X. Shen, X. Wang, F. Sun, Journal of Materials Processing Technology, 265, 1-11 (2019)

9. P.W. May, M.N.R. Ashfold, Y.A Mankelevich, Journal of Applied Physics, 101, № 5, 1-9 (2007)

10. J. Weng, Vacuum, 147, 134-142, (2018)

11. T. Teraji, S. Mitani, T. Ito, physica status solidi a, 198, 395-406 (2003)

12. A.T. Collins, Diamond and Related Materials, 1, № 5-6, 457-469 (1992)

13. B. Willems, A. Tallaire, J. Achard, Diamond and Related Materials, 41, 25-33 (2014)

14. Available online: https://assets.thermofisher.com/TFS-Assets/CAD/ApplicationNotes/D19504 .pdf (accessed on 21 May 2020).

15. A.V. Nozhkina, A.I. Laptev, A.A. Ermolaev, Sverkhtverdye Materialy, 5, 36-39 (2002)

16. A.V. Elyutin, A.A. Ermolaev, A.I. Laptev, A.V.Manukhin, Doklady Physics, 47, I. № 9, 61-65 (2002)

17. A.V. Elyutin, A.I. Laptev, A.V. Manukhin, D.S. Sannikov, L.M. Kryukova, Doklady Chemistry, 378, I. 4-6, 160-164 (2001) 